Forensic toxicology

\title{
VALIDATED QUANTITATIVE SIMULTANEOUS DETERMINATION OF COCAINE, OPIATES AND AMPHETAMINES IN SERUM BY U-HPLC COUPLED TO TANDEM MASS SPECTROMETRY
}

\author{
N. Dubois', B. Debrus ${ }^{2,3}$, Ph. Hubert ${ }^{2,3}$, C. Charlier ${ }^{3,3}$ \\ Key words: Cocaine, opiates, amphetamines, UHPLC-MS/MS method, validation, total error
}

\section{ABSTRACT}

Simultaneous determination of cocaine, opiates and amphetamines in serum by ultra-high performance liquid chromatography coupled to tandem mass spectrometry (UHPLC-MSTMS) allowed to replace favourably gas chromatography coupled to mass spectrometry (GC-MS) used until now in our laboratory. It had to answer to accreditation demand according to Belgian Accreditation (Belac).

Twenty-one deutereted internal standards were added to $500 \mu \mathrm{L}$ of serum. Sample pre-treatment consisted of solid-phase extraction using Oasis MCX cartridges $1 \mathrm{~mL}, 30 \mathrm{mg}$ (Waters, Zellik, Belgium). Chromatographic separation was done on an Acquity HSS T3 column $(2.1 \times 100 \mathrm{~mm}, 1.8 \mu \mathrm{m}$,

1 Service de Toxicologie Clinique,

Médicolégale, de l'Environnement et en Entreprise,

CHU Sart-Tilman, Liège, Belgique.

2 Service de Chimie Analytique.

Département de Pharmacie,

Université de Liège, Belgique.

3 CIRM, Universitê de Liège, Belgique.

Address for Correspondence

N. Dubois

Service de Toxicologie Clinique, Médicolégale.

de l'Environnement et en Entreprise.

CHU Sart-Tilman B35, Tour $11,+5$

B-4000 Liège

Belgique

Tél.: +3243667680

Fax: +3243668889

E-mail: nathalie.dubois@chu.ulg.ac.be
Waters). Mobile phase consisted of $\mathrm{pH} 3$ ammonium formate buffer and of methanol adjusted to $\mathrm{pH} 3$ with formic acid, Compounds were next analysed by tandem mass spectrometry operated in the multiple reaction monitoring (MRM) mode. The method was validated using total error approach.

Twenty-seven drugs were separated in 19 minutes. The linearity of the method was acceptable in the validated range of concentrations. The bias and the relative standard deviations for repeatability and intermediate precision were acceptable. Lower and upper $\beta$-expectation tolerance limits did not exceed the acceptance limits of $20 \%$ for concentrations upper than $20 \% \mathrm{~g} / \mathrm{L}$ and $50 \%$ for concentrations lower than 20ug/L.The limits of quantitation were lower than 7 ug/L for all compounds.

\section{INTRODUCTION}

The use of drugs of abuse is increasing worldwide and causing serious social problems. About $3.9 \%$ of European adults would have used cocaine last year (1). Benzoylecgonine is one of the major metabolites of cocaine formed by either spontaneous hydrolysis or by hepatic carboxyesterase enzymes. When cocaine is co-administered with alcohol, cocaethylene is formed in the body (2). About $3.1 \%$ of European adults would have used ecstasy and related compounds last year (1). During the last two decades, the abuse of 3,4-methylenedioxymethamphetamine (MDMA) - prototype of designer drugs - has increased considerably. Some homologous cornpounds with similar effects, such as 
methylenedioxyamphetamine (MDA), methylenedioxyethylamphetamine (MDEA) and $\mathrm{N}$-methyl-1(3,4-methylenedioxyphenyl)-2-butanamine (MBDB) have also appeared on the market, but are less often used (3). The number of opioid users is estimated at between 1.2 and 1.5 millions in Europe and about 650000 of them would have received substitution treatment in 2007 . Heroin accounts for the greatest share of morbidity and mortality related to drug use in the European Union (1). Once in the body, heroin is very rapidly converted by deacetylation using a plasmatic esterase to form 6-acetylmorphine (6-MAM), which is hydrolyzed into morphine with a hepatic esterase. Morphine is quickly converted to its principal metabolite, morphine-3-glucuronide (M3G) and somewhat more slowly to smaller amounts of morphine-6-glucuronide (M6G). According to the report of the European Monitoring Centre for Drugs and Drug Addiction published in November 2009 (1), polydrug patterns are today the norm in Europe and the combined use of different substances is responsible for, or complicates, most of the problems Europe faces.

To our knowledge only a few methods allowed simultaneous determination of drugs of abuse in biological fluids $(4,5,6)$. The aim of this method was to allow simultaneously the quantitative determination of cocaine and two of its metabolites - benzoylecgonine and cocaethylene - of amphetamines commonly used in Europe - amphetamine, methamphetamine, MDMA, $M D A$ and $M B D B$ - and of heroin and its metabolites 6-MAM, morphine, M3G and M6G. Others opioids were added to the method: hydromorphone, pholcodine, codeine, dihydrocodeine, hydrocodone, oxycodone, ethylmorphine and drugs used for substitution treatment: methadone and its metabolite 2-ethylidine1,5-dimethyl-3,3-diphenidylpyrrolidine (EDDP) and buprenorphine and its metabolite norbuprenorphine. Naloxone and naltrexone, two narcotic antagonists, were also quantified. Gas chromatography coupled to mass spectrometry (GC-MS) had been widely used for many years, $(7,8)$ but, recently, liquid chromatography coupled to tandem mass spectrometry (LC-MS/MS) was becoming increasingly important for the quantitative determination of drugs of abuse $(2-6,9)$. The technology we had chosen for the quantification of 27 compounds was the ultra-high performance liquid chromatography coupled to tandem mass spectrometry (UHPLC-MS/MS). It replaced favourably GC-MS techniques used until now in our laboratory.

\section{MATERIALS AND METHODS}

\section{Chemicals and reagents}

Reference materials for all compounds and internal standards were purchased from LGC Promochem (Molsheim, France). All standards had a degree of purity upper than $99 \%$.

Methanol, water and formic acid (LC-MS grade) were purchased from Biosolve (Valkenswaard, the Netherlands); citric acid and ammonium formate, at least of analytical grade, were purchased from Sigma (Steinheim, Germany), ammonia from VWR. Int. (Leuven, Belgium). Solid phase extraction (SPE) cartridges, Oasis $\mathrm{MCX}$ (30mg, $1 \mathrm{~mL}$ ), were obtained from Waters (Zellik, Belgium).

\section{Stock solutions and standards}

For the preparation of the stock solution of internal standard, commercial solutions of cocaine- $d_{3}$, benzoylecgonine- $d_{3}$, cocaethylene- $d_{3}$, morphine- $d_{3^{\prime}}$, codeine$d_{3^{\prime}}, 6$-acetylmorphine- $d_{3^{\prime}}$, heroin- $d_{9^{\prime}}$ oxycodone- $d_{3^{\prime}}$, hydromorphone- $d_{3^{\prime}}$ dihydrocodeine- $d_{6^{*}}$, hydrocodone- $d_{3^{\prime}}$ methadone- $d_{3^{\prime}}$ EDDP- $d_{3^{\prime}}$ morphine-6-glucuronide- $d_{3^{\prime}}$ morphine-3glucuronide- $d_{3}$, amphetamine- $d_{g^{\prime}}$ methamphetamine- $d_{8}$, MDMA- $d_{5}$, MDA- $d_{5}$, MBDB- $d_{5}$ and buprenorphine- $d_{4}$ were diluted in methanol. Concentration of the internal standard stock solution was $1 \mathrm{mg} / \mathrm{L}$ for all compounds except for buprenorphine- $\mathrm{d}_{4}$ $(100 \mu \mathrm{g} / \mathrm{L})$.

Calibration standards and validation standards were prepared by spiking drug-free serum with stock solution containing all the compounds. The first group of analytes was constituted of amphetamine (AMP), methamphetamine (MAMP), MDMA, MDA, MBDB, 6-acetylmorphine (6MAM), morphine (MOR), oxycodone (OCOD) and hydromorphone (HMOR); the second group of cocaine (COC), benzoylecgonine (BZE), cocaethylene (COCET), codeine (COD), norcodeine (NCOD), 6-acetylcodeine (6ACOD), dihydrocodeine (DHCOD), hydrocodone (HCOD), ethylmorphine (EMOR), pholcodine (PHOL), methadone (METHA), EDDP, M3G, M6G, naloxone and naltrexone and the third one of buprenorphine (BUP) and norbuprenorphine (NBUP). Group constitution depends on the expected analyte concentration. Calibration standards were prepared to obtain final concentration of $2,5,10$, 20,40 and $80 \mu \mathrm{g} / \mathrm{L}$ for group $1 ; 10,25,50,100,200$ and $400 \mu \mathrm{g} / \mathrm{L}$ for group $2 ; 1,2.5,5,10,20$ and $40 \mu \mathrm{g} / \mathrm{L}$ for group 3 ; they were analyzed in duplicate for three days and were used to establish the calibration curves 
(response function). Validation standards were prepared at the concentrations of $1,2,4,6,10,30,60$ and $100 \mu \mathrm{g} / \mathrm{L}$ for group $1,5,10,30,150,300$ and $500 \mu \mathrm{g} / \mathrm{L}$ for group 2 and $0.5,1,3,15,30$ and $50 \mu \mathrm{g} / \mathrm{L}$ for group 3; they were analyzed in triplicate for three days and were used to estimate the validation parameters and thus the method limits. An extract of drug-free serum was also prepared for each run.

\section{Sample pre-treatment}

Fifty $\mu \mathrm{L}$ of internal standard stock solution were added to $500 \mu \mathrm{L}$ of serum, then it was acidified with $500 \mu \mathrm{L} \mathrm{HCl} 0.15 \mathrm{~N}$. Oasis $\mathrm{MCX}(30 \mathrm{mg}, 1 \mathrm{~mL})$ cartridges were used for solid phase extraction. They were conditioned with 2 times $1 \mathrm{~mL}$ of methanol, $1 \mathrm{~mL}$ of water and 2 times $1 \mathrm{~mL}$ of citric acid $10 \mathrm{mM}(\mathrm{pH} 3.0)$. The SPE cartridge was not allowed to run dry during the conditioning step. The acidified sample was loaded onto the columns and the flow was kept at approximately $1 \mathrm{~mL}$ $\min (9)$. Then, cartridge was washed with $500 \mu \mathrm{L}$ of formic acid $2 \%$ and dried at maximal vacuum for $1 \mathrm{~min}$. Analytes were eluted with $1 \mathrm{~mL}$ ammonia in methanol solution 5:95 (v/v). The eluate was evaporated to dryness under gentle nitrogen flow and reconstituted with $100 \mu \mathrm{L}$ of a mixture of ammonium formate $5 \mathrm{mM}$ $(\mathrm{pH} 3)$ and methanol adjusted to $\mathrm{pH} 3$ with formic acid 90:10 (v/v). Ten $\mu \mathrm{L}$ were injected to the column.

\section{Instrumentation}

Analysis was performed on an UPLC Acquity coupled to a tandem mass spectrometer Quattro Premier (Waters, Zellik, Belgium). The chromatographic separation was done on an Acquity High Strength Silica HSST3 column ( $100 \times 2.1 \mathrm{~mm}$ i.d, particle size $1.8 \mu \mathrm{m}$, Waters) equipped with an on-line filter at $40^{\circ} \mathrm{C}$. Gradient elution was performed at a constant flow of $0.5 \mathrm{~mL} /$ min. using a mixture of $5 \mathrm{mM}$ ammonium formate in

Table 1 - UPLC elution gradient, $A=$ ammonium formate $5 \mathrm{mM} \mathrm{pH} \mathrm{3,} \mathrm{B} \mathrm{=} \mathrm{methanol} \mathrm{pH} 3$

\begin{tabular}{ccc}
\hline Time $(\min )$ & A\% & $B \%$ \\
\hline 0.0 & 100.0 & 0.0 \\
1.0 & 100.0 & 0.0 \\
2.0 & 92.5 & 7.5 \\
5.5 & 89.0 & 11.0 \\
16.0 & 10.0 & 90.0 \\
17.0 & 10.0 & 90.0 \\
18.0 & 100.0 & 0.0 \\
19.0 & 100.0 & 0.0 \\
\hline
\end{tabular}

water $(\mathrm{pH} 3)$ and methanol adjusted to $\mathrm{pH} 3$ with formic acid, as described in Table 1.

After chromatographic separation, compounds were analyzed in the tandem mass spectrometer operated in the positive electrospray mode at $1.0 \mathrm{kV}$, at a source temperature of $120^{\circ} \mathrm{C}$ and at a desolvatation temperature of $350^{\circ} \mathrm{C}$. The collision gas flow was set at $50 \mathrm{~L} / \mathrm{h}$ and the desolvatation gas flow was set at $800 \mathrm{~L} / \mathrm{h}$ The MS method was divided into 5 functions depending on the retention times of the analytes. Two multiple reaction monitoring (MRM) were studied by molecule for identification and quantification when molecule fragmentation allowed it (see Table 2).

\section{Method validation}

According to ISO17025 and the guidelines of the French Society of Pharmaceutical Sciences and Techniques (SFSTP), the present method was fully validated using total error approach $(10,11,12)$. The e-noval software V3.0 (Arlenda, Liège, Belgium) was used to compute all validation results and to build the accuracy profiles.

\section{RESULTS}

Elution was carried out using a segmented gradient of 19 minutes. To avoid column contamination with matrix highly retained compounds, a one minute duration step, with mobile phase of high elution strength, was kept at the end of the gradient (between 16 and 17 minutes) before initial conditions re-equilibration. The 27 compounds were well separated, with retention times from 2.5 to $13.5 \mathrm{~min}$. A chromatogram of an extracted spiked serum is presented in Figure 1.

The response function is, within the range, the existing relationship between the response (signal) and the concentration of the analyte in the sample (11). It was build from the calibration standards. The response function was a linear regression weighted or not, or a quadratic regression, weighted or not, depending on the analyte.

The linearity is the method ability to obtain results directly proportional to the concentrations of the analyte in the sample (11). The method presents a good linearity in the validated range for each compound.

The trueness expresses the closeness of agreement between the mean value obtained from the validation standards and the value which is accepted either as a conventional true value or an accepted reference value. 
Table 2 - Retention times and MRM transitions of each analyte and internal standard (IS)

\begin{tabular}{|c|c|c|c|c|c|c|c|}
\hline Compound & Internal std & $\begin{array}{l}\text { Ret. time } \\
\text { (min.) }\end{array}$ & $\begin{array}{c}\text { Cone } \\
\text { voltage }(V)\end{array}$ & MRM1 & $\begin{array}{c}\text { Collision } \\
\text { energy } \\
\text { (V) } \\
\end{array}$ & MRM2 & $\begin{array}{c}\text { Collision } \\
\text { energy } \\
\text { (V) }\end{array}$ \\
\hline $\begin{array}{l}\text { M3G- } d_{3} \\
\text { M6G-d }\end{array}$ & - & $\begin{array}{l}2.86 \\
3.29\end{array}$ & 39 & $465.10>289.20$ & 33 & - & - \\
\hline $\begin{array}{l}\text { M3G } \\
\text { M6C }\end{array}$ & $\begin{array}{l}\text { M3G- } d_{3} \\
\text { M6C-d }\end{array}$ & 2.883 .31 & 39 & $462.25>462.25$ & 12 & $462.25>286.25$ & 33 \\
\hline Pholcodine & Morphine- $d_{3}$ & 3.00 & 35 & $399.20>399.20$ & 10 & $399.2>114.10$ & 31 \\
\hline Morphine- $d_{3}$ & - & 3.40 & 35 & $289.05>201.20$ & 28 & - & - \\
\hline Morphine & Morphine- $d_{3}$ & 3.41 & 35 & $286.10>286.10$ & 12 & $286.10>201.10$ & 29 \\
\hline Hydromorphone- $d_{3}$ & - & 4.18 & 35 & $289.10>185.10$ & 28 & - & - \\
\hline Hydromorphone & Hydromorphone- $d_{3}$ & 4.20 & 35 & $286.10>286.10$ & 12 & $286.10>185.00$ & 31 \\
\hline Norcodeine & Codeine- $d_{3}$ & 6.42 & 30 & $286.20>286.25$ & 10 & $286.20>268.15$ & 20 \\
\hline Dihydrocodeine- $d_{6}$ & - & 6.42 & 30 & $308.15>202.15$ & 32 & - & - \\
\hline Dihydrocodeine & Dihydrocodéine- $d_{6}$ & 6.44 & 30 & $302.15>302.15$ & 9 & $302.15>199.15$ & 32 \\
\hline Codeine $-d_{3}$ & - & 6.50 & 35 & $303.15>215.15$ & 25 & - & \\
\hline Codeine & Codeine- $d_{3}$ & 6.52 & 35 & $300.15>300.15$ & 13 & $300.15>215.15$ & 25 \\
\hline Naloxone & Codeine- $d_{3}$ & 6.62 & 27 & $328.10>328.30$ & 8 & $328.10>114.10$ & 20 \\
\hline Oxycodone- $d_{3}$ & - & 7.01 & 28 & $319.10>301.25$ & 18 & - & - \\
\hline Oxycodone & Oxycodone- $d_{3}$ & 7.03 & 28 & $316.25>298.30$ & 19 & $316.25>241.25$ & 30 \\
\hline Hydrocodone- $d_{3}$ & - & 7.33 & 35 & $303.15>215.15$ & 25 & - & - \\
\hline Hydrocodone & Hydrocodone- $d_{3}$ & 7.35 & 39 & $300.15>300.15$ & 13 & $300.15>215.15$ & 25 \\
\hline Amphetamine- $d_{\theta}$ & - & 6.85 & 16 & $144.00>127.10$ & 8 & - & - \\
\hline Amphetamine & Amphetamine- $d_{8}$ & 6.87 & 16 & $136.00>119.10$ & 8 & $136.00>91.00$ & 16 \\
\hline Methamphetamine- $d_{8}$ & - & 7.34 & 19 & $158.00>124.05$ & 11 & - & - \\
\hline Methamphetamine & Methamphetamine- $d_{8}$ & 7.36 & 19 & $150.00>119.10$ & 11 & $150.00>91.00$ & 20 \\
\hline Naltrexone & Codeine- $d_{3}$ & 7.42 & 27 & $342.15>342.15$ & 10 & $342.15>324.15$ & 21 \\
\hline MDA- $d_{5}$ & - & 7.47 & 15 & $185.00>168.05$ & 11 & - & - \\
\hline MDA & MDA-d ${ }_{5}$ & 7.49 & 15 & $180.00>163.10$ & 11 & $180.00>105.00$ & 23 \\
\hline 6-MAM-d & - & 7.61 & 39 & $331.20>211.10$ & 24 & - & - \\
\hline 6-MAM & 6-MAM-d ${ }_{3}$ & 7.63 & 39 & $328.20>211.10$ & 24 & $328.20>165.10$ & 40 \\
\hline MDMA- $d_{5}$ & - & 7.70 & 20 & $199.05>165.05$ & 13 & - & - \\
\hline MDMA & MDMA- $d_{5}$ & 7.72 & 20 & $194.05>163.05$ & 13 & $194.05>105.00$ & 24 \\
\hline Ethylmorphine & Codeine- $d_{3}$ & 8.19 & 35 & $314.25>314.30$ & 12 & - & - \\
\hline Benzoylecgonine- $d_{3}$ & - & 8.88 & 26 & $293.00>171.10$ & 18 & - & - \\
\hline Benzoylecgonine & Benzoylecgonine- $d_{3}$ & 8.90 & 26 & $290.20>168.15$ & 18 & $290.20>105.10$ & 30 \\
\hline MBDB- $d_{5}$ & - & 8.98 & 18 & $213.10>179.10$ & 11 & - & - \\
\hline MBDB & MBDB- $d_{5}$ & 9.00 & 18 & $208.20>177.15$ & 11 & $208.20>135.10$ & 11 \\
\hline Acetylcodeine & Codeine- $d_{3}$ & 9.43 & 40 & $342.30>342.30$ & 11 & $342.30>225.25$ & 28 \\
\hline Cocaine- $d_{3}$ & - & 9.55 & 27 & $307.10>185.10$ & 21 & - & - \\
\hline Cocaine & Cocaine- $d_{3}$ & 9.57 & 27 & $304.20>182.15$ & 21 & $304.20>82.15$ & 32 \\
\hline Cocaethylene- $d_{3}$ & - & 10.43 & 30 & $321.15>199.15$ & 19 & - & $\cdot$ \\
\hline Cocaethylene & Cocaethylene- $d_{3}$ & 10.45 & 30 & $318.25>196.20$ & 19 & $318.25>82.15$ & 30 \\
\hline Buprenorphine- $d_{4}$ & - & 11.98 & 60 & $472.20>400.20$ & 42 & - & - \\
\hline Buprenorphine & Buprenorphine- $d_{4}$ & 12.00 & 60 & $468.15>414.15$ & 36 & $468.15>396.15$ & 42 \\
\hline Norbuprenorphine & Buprenorphine- $d_{4}$ & 11.14 & 60 & $414.40>101.10$ & 35 & $414.40>83.10$ & 65 \\
\hline$E D D P-d_{3}$ & - & 11.57 & 35 & $281.15>249.20$ & 24 & - & - \\
\hline EDDP & $E D D P-d_{3}$ & 11.59 & 35 & $278.15>249.20$ & 24 & $278.15>234.10$ & 32 \\
\hline Methadone- $d_{3}$ & - & 12.82 & 25 & $313.20>268.20$ & 15 & - & - \\
\hline Methadone & Methadone- $d_{3}$ & 12.84 & 25 & $310.20>310.20$ & 5 & $310.20>265.20$ & 15 \\
\hline
\end{tabular}




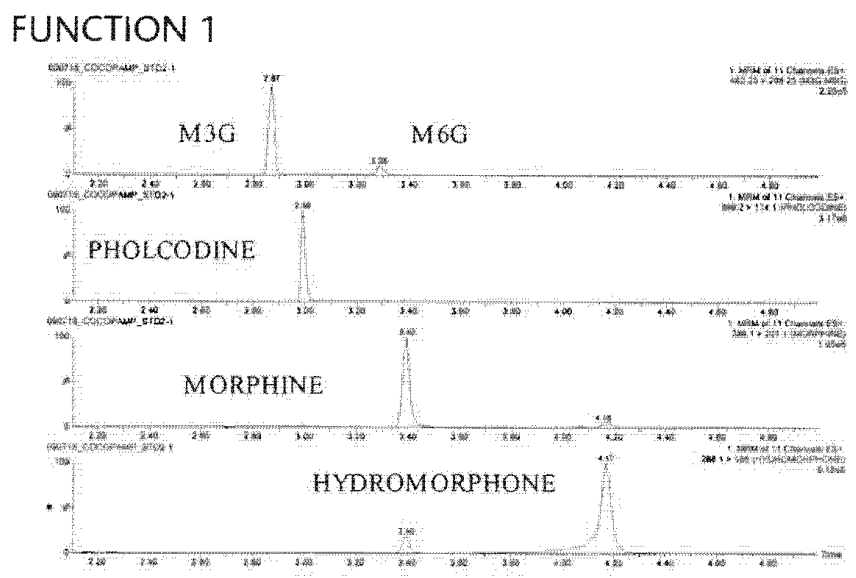

\section{FUNCTION 2}

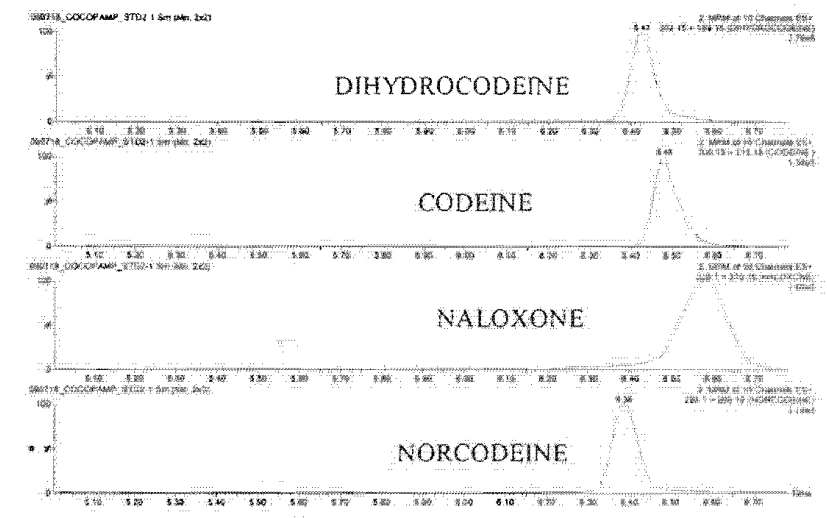

\section{FUNCTION 3}

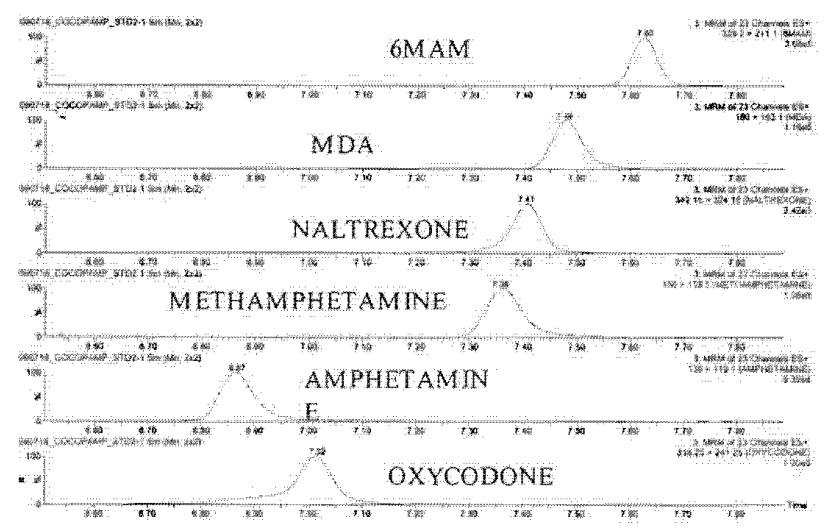

\section{FUNCTION 4}

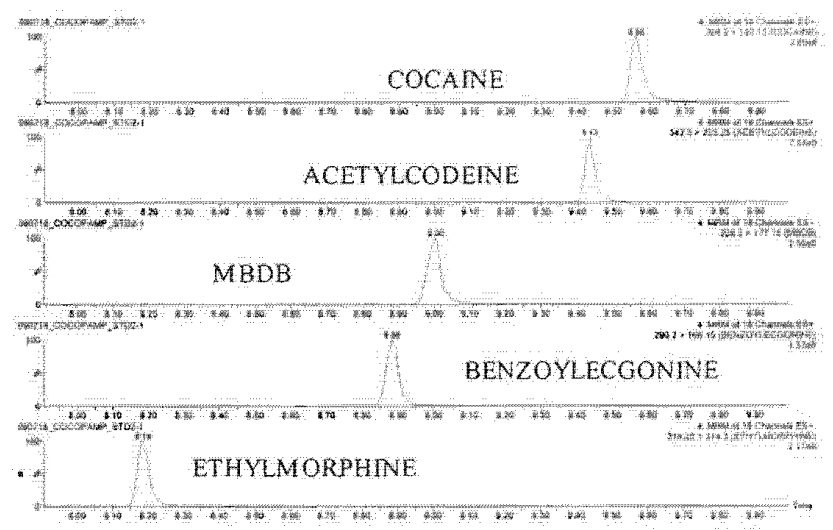

\section{FUNCTION 5}

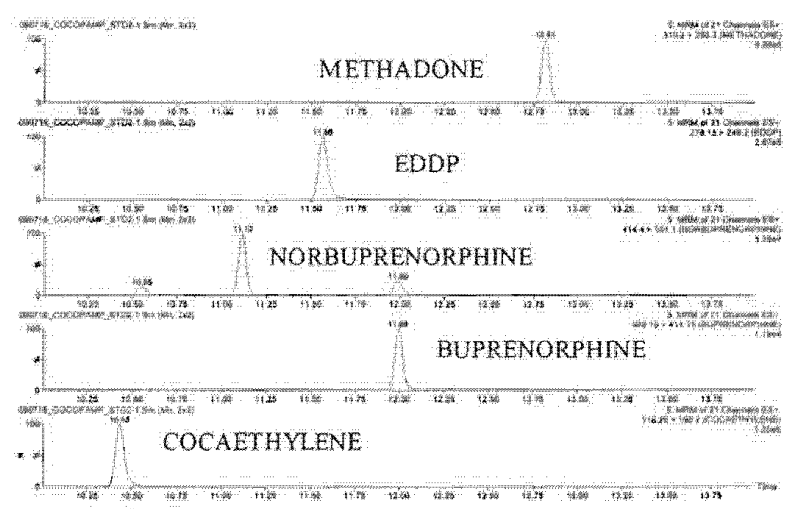

Figure 1-Chromatogram example of an extracted spiked serum

Trueness is expressed in terms of relative bias (systematic error) (11). Trueness was acceptable for all compounds, since the relative biases were always smaller than $19 \%$ for concentrations lower than $20 \mu \mathrm{g} / \mathrm{L}$ and than $12 \%$ for concentrations upper than $20 \mu g / L$. Results are presented in Table2.

The precision was determined by computing the Relative Standard Deviations (RSDs) for repeatability and intermediate precision at each concentration level of the validation standards (13). They did not exceed $20 \%$ for concentrations lower than $20 \mu \mathrm{g} / \mathrm{L}$ and $8 \%$ for concentrations upper than $20 \mu \mathrm{g} / \mathrm{L}$ for repeatability; they did not exceed $30 \%$ for concentrations lower than $20 \mu \mathrm{g} / \mathrm{L}$ and $15 \%$ for concentrations upper than $20 \mu \mathrm{g} / \mathrm{L}$ for intermediate precision. RSDs are presented in Table2.

The uncertainty characterizes the dispersion of the values that could reasonably be attributed to the 
Table 3 Trueness, precision, uncertainty of measurement, lower- and upper- limit of quantitation Group 1

\begin{tabular}{|c|c|c|c|c|c|c|c|c|c|c|}
\hline & $\begin{array}{l}\text { Target } \\
\text { conc. } \\
\mu g / L\end{array}$ & AMP & MAMP & MOMA & MDA & $M B O B$ & GMAM & MOR & $O C O D$ & HMOR \\
\hline \multirow{8}{*}{$\begin{array}{l}\text { Trueness } \\
\text { Relative bias (\%) }\end{array}$} & 1.0 & 2.77 & 0.00 & 11.51 & 15.34 & 2.15 & 18.43 & -2.58 & 7.87 & -12.18 \\
\hline & 2.0 & -3.87 & -9.79 & -2.11 & 1.90 & -3.98 & 1.50 & -8.21 & 1.79 & -8.72 \\
\hline & 4.0 & 1.70 & -0.13 & 1.45 & 1.39 & 0.06 & 1.77 & -3.58 & 3.42 & -2.42 \\
\hline & 6.0 & 3.67 & 0.58 & 1.63 & 0.51 & 4.30 & 3.21 & .0 .40 & 6.26 & -2.14 \\
\hline & 10.0 & 0.62 & 2.85 & 1.22 & -2.27 & 2.82 & -1.79 & -0.41 & 3.70 & -3.30 \\
\hline & 30.0 & 6.81 & 6.05 & 5.78 & 5.52 & 4.63 & 5.25 & 3.43 & 2.38 & 6.83 \\
\hline & 60.0 & 8.81 & 4.47 & 4.85 & 7.32 & 4.90 & 4.28 & 7.36 & 2.14 & 2.32 \\
\hline & 100.0 & 4.87 & 2.51 & 6.40 & 6.71 & 2.69 & 5.26 & 9.24 & -0.85 & 6.52 \\
\hline \multirow{8}{*}{$\begin{array}{l}\text { Intra-assay precision } \\
\text { Repeatability } \\
(\mathrm{RSD} \%)\end{array}$} & 1.0 & 10.65 & 6.69 & 2.86 & 6.59 & 5.71 & 7.19 & 10.87 & 1.88 & 7.24 \\
\hline & 2.0 & 3.15 & 4.14 & 2.92 & 2.34 & 2.85 & 4.50 & 5.90 & 5.65 & 5.57 \\
\hline & 4.0 & 5.09 & 4.50 & 2.58 & 5.11 & 3.52 & 2.47 & 3.37 & 6.15 & 8.39 \\
\hline & 6.0 & 6.78 & 4.09 & 4.73 & 5.44 & 4.49 & 3.64 & 3.47 & 0.56 & 3.90 \\
\hline & 10.0 & 2.17 & 2.84 & 2.19 & 3.34 & 4.01 & 2.66 & 2.34 & 1.35 & 1.62 \\
\hline & 30,0 & 2.33 & 4.77 & 3.04 & 3.41 & 3.12 & 1.67 & 1.62 & 1.61 & 1.95 \\
\hline & 60.0 & 1.89 & 1.09 & 1.86 & 2.30 & 2.41 & 1.81 & 2.05 & 0.77 & 1.11 \\
\hline & 100.0 & 2.83 & 2.21 & 3.78 & 4.19 & 3.69 & 3.56 & 5.38 & 2.24 & 5.23 \\
\hline \multirow{8}{*}{$\begin{array}{l}\text { Inter-assay precision } \\
\text { Intermediate } \\
\text { precision (RSD\%) }\end{array}$} & 1.0 & 14.02 & 41.92 & 14.43 & 10.89 & 26.99 & 11.99 & 48.71 & 9.78 & 97,67 \\
\hline & 2.0 & 8.50 & 22.74 & 4.23 & 10.72 & 19.62 & 4.50 & 33.47 & 8.73 & 50.73 \\
\hline & 4.0 & 5.18 & 10.68 & 2.58 & 6.80 & 8.74 & 2.87 & 10.72 & 7.07 & 34.91 \\
\hline & 6.0 & 8.35 & 11.56 & 4.73 & 9.10 & 12.66 & 3.64 & 8.25 & 10.19 & 22.42 \\
\hline & 10.0 & 10.07 & 7.90 & 7.06 & 8.65 & 12.67 & 5.31 & 5.27 & 7.13 & 15.63 \\
\hline & 30.0 & 5.43 & 4,77 & 4.52 & 6.47 & 7.25 & 1.76 & 5.99 & 5.36 & 4.23 \\
\hline & 60.0 & 4.95 & 5.70 & 2.04 & 4.32 & 2.73 & 1.81 & 3.08 & 3.50 & 6.65 \\
\hline & 100,0 & 5.67 & 10.28 & 8.53 & 14.87 & 13.07 & 5.44 & 6.32 & 6.57 & 8.28 \\
\hline \multirow{8}{*}{$\begin{array}{l}\text { Uncertainty } \\
\text { Relative expended } \\
\text { uncertainty }(\%)\end{array}$} & 1.0 & 30.79 & 96.59 & 33.21 & 24.37 & 62.10 & 26.85 & 112.00 & 22.51 & 225.4 \\
\hline & 2.0 & 19.41 & 52.38 & 9.38 & 24.66 & 45.23 & 9.49 & 77.10 & 19.45 & 117.0 \\
\hline & 4.0 & 10.96 & 24.29 & 5.44 & 14.95 & 19.91 & 6.20 & 24.54 & 15.25 & 80.22 \\
\hline & 6.0 & 18.20 & 26.41 & 9.98 & 20.37 & 28.94 & 7.67 & 18.78 & 23.53 & 51.65 \\
\hline & 10.0 & 23.17 & 18.04 & 16.17 & 19.73 & 29.02 & 12.07 & 11.97 & 16.42 & 36.07 \\
\hline & 30.0 & 12.34 & 10.05 & 10.02 & 14.60 & 16.47 & 3.76 & 13.75 & 12.29 & 9.60 \\
\hline & 60.0 & 11.28 & 13.11 & 4.39 & 9.74 & 5.89 & 3.82 & 6.85 & 8.04 & 15.08 \\
\hline & 100.0 & 12.83 & 23.67 & 19.37 & 34.14 & 29,97 & 12,11 & 13.81 & 15.01 & 18.49 \\
\hline $\operatorname{LQL}(\mu \mathrm{g} / \mathrm{L})$ & & 1.0 & 2.6 & 1.0 & 1.0 & 2.0 & 1.0 & 3.2 & 1.0 & 7.3 \\
\hline UQL $(\mu g / L)$ & & 100.0 & 70.6 & 89.4 & 66.5 & 78.6 & 100.0 & 98.5 & 100.0 & 85.8 \\
\hline
\end{tabular}

Legend: $A M P=$ amphetamine, $M A M P=$ methamphetamine, $6 M A M=6$-acetylmorphine, $M O R=$ morphine, $O C O D=0 x y c o d o n e, H M O R=$ hydromorphone 
Group 2

\begin{tabular}{|c|c|c|c|c|c|c|c|c|c|c|c|}
\hline & $\begin{array}{l}\text { Target } \\
\text { conc. } \\
u g / L\end{array}$ & $\mathrm{COC}$ & $\overline{B Z E}$ & COCET & $\mathrm{COD}$ & NCOD & 6ACOD & DHCOD & $\mathrm{HCOD}$ & EMOR & $\mathrm{PHOL}$ \\
\hline \multirow{6}{*}{$\begin{array}{l}\text { Trueness } \\
\text { Relative bias }(\%)\end{array}$} & 5.0 & 6.75 & 3.13 & -2.09 & 14.49 & 12.25 & -2.95 & -28.38 & 5.77 & 4.86 & 16.77 \\
\hline & 10.0 & -6.26 & -5.98 & -7.29 & 2.84 & -3.13 & -6.44 & -12.21 & 2.41 & -2.80 & 6.32 \\
\hline & 30.0 & -5.60 & -8.63 & -2.24 & 1.12 & -3.46 & 2.35 & 4.79 & -3.67 & 1.25 & 8.17 \\
\hline & 150.0 & -1.32 & -3.24 & 0.16 & 2.57 & -2.67 & -6.54 & 9.18 & 0.68 & 3.92 & -0.85 \\
\hline & 300 & 3.16 & 2.36 & 2.04 & 3.96 & -3.51 & $=$ & 6.62 & -0.14 & - & - \\
\hline & 500 & 4.23 & 1.46 & -2.26 & 0.15 & - & - & 2.22 & -1.27 & - & - \\
\hline \multirow{6}{*}{$\begin{array}{l}\text { Intra-assay } \\
\text { precision } \\
\text { Repeatability (RSD\%) }\end{array}$} & 5.0 & 8.74 & 9.17 & 7.04 & 3.11 & 5.31 & 10.26 & 4.61 & 2.59 & 6.09 & 12.91 \\
\hline & 10.0 & 3.83 & 5.56 & 2.50 & 2.75 & 2.99 & 5.83 & 3.72 & 3.21 & 6.55 & 6.49 \\
\hline & 30.0 & 3.44 & 3.18 & 3.49 & 4.58 & 6.59 & 4,95 & 4,30 & 5.79 & 4.21 & 3.84 \\
\hline & 150.0 & 4.03 & 4.09 & 5.88 & 2.11 & 7.62 & 6.38 & 0.72 & 1.22 & 5.15 & 4.85 \\
\hline & 300.0 & 3.55 & 3.62 & 2.85 & 1.51 & 6.40 & - & 0.89 & 3.04 & $\cdots$ & - \\
\hline & 500.0 & 4.52 & 4.02 & 2.44 & 2.29 & - & - & 2.76 & 3.23 & - & - \\
\hline \multirow{6}{*}{$\begin{array}{l}\text { Inter-assay } \\
\text { precision } \\
\text { Intermediate precision } \\
\text { (RSD\%) }\end{array}$} & 5.0 & 14.93 & 29.31 & 12.76 & 14.5 & 24.00 & 10.26 & 4.61 & 4.71 & 27.51 & 14.35 \\
\hline & 10.0 & 4.15 & 11.36 & 4.18 & 6.79 & 13.03 & 5.83 & 4.35 & 4.81 & 10.90 & 7.13 \\
\hline & 30.0 & 3.65 & 3.18 & 6.26 & 4.58 & 7.19 & 5.03 & 5.48 & 5.79 & 4.43 & 6.19 \\
\hline & 150.0 & 4.90 & 4.36 & 5.88 & 5.60 & 9.33 & 6.38 & 1.16 & 1.55 & 6.94 & 5.13 \\
\hline & 300.0 & 3.55 & 3.62 & 3.40 & 5.42 & 6.40 & - & 1.42 & 3.68 & - & - \\
\hline & 500,0 & 4.77 & 5.59 & 2.44 & 8.42 & - & - & 7.64 & 3.90 & $\therefore$ &.- \\
\hline \multirow{6}{*}{$\begin{array}{l}\text { Uncertainty } \\
\text { Relative expended } \\
\text { uncertainty }(\%)\end{array}$} & 5.0 & 33.48 & 67.14 & 28.72 & 33.35 & 55.24 & 21.63 & 9.72 & 10.59 & 63.28 & 30.83 \\
\hline & 10.0 & 8.88 & 25.71 & 9.37 & 15.47 & 29.96 & 12.30 & 9.41 & 10,68 & 24.41 & 15.29 \\
\hline & 30.0 & 7.79 & 6.87 & 14.09 & 9.66 & 15.41 & 10.63 & 11.98 & 12.21 & 9.44 & 13.82 \\
\hline & 150,0 & 10.67 & 9.31 & 12,40 & 12.77 & 20.32 & 13.78 & 2.59 & 3.38 & 15.28 & 11.00 \\
\hline & 300.0 & 7.49 & 7.62 & 7.37 & 12.44 & 13.48 & " & 3.17 & 8.00 & - & - \\
\hline & 500.0 & 10.15 & 12.33 & 5.15 & 19.31 & - & - & 17.46 & 8.48 & - & - \\
\hline $\operatorname{LQL}(\mu \mathrm{g} / \mathrm{L})$ & & 5.0 & 6.9 & 5.0 & 5.0 & 7.2 & 5.0 & 5.0 & 5.0 & 6.8 & 5.0 \\
\hline$U Q L(\mu g / L)$ & & 500.0 & 500.0 & 500.0 & 500.0 & 300.0 & 150.0 & 500.0 & 500.0 & 150.0 & 150.0 \\
\hline
\end{tabular}

Legend: $C O C=$ cocaine, $B Z E=$ benzoylecgonine, $C O C E T=$ cocaethylene, $C O D=$ codeine, $N C O D=$ norcodeine, $6 A C O D=6$-aCetylcodeine, DHCOD = dihydrocodeine, $\mathrm{HCOD}=$ hydrocodone, $\mathrm{EMOR}=$ ethylmorphine, $\mathrm{PHOL}=$ pholcodine.

Group 2 (continued) and group 3

\begin{tabular}{|c|c|c|c|c|c|c|c|c|c|c|}
\hline & $\begin{array}{l}\text { Target } \\
\text { conc. } \\
\text { Hg/L }\end{array}$ & METHA & EDDP & $\mathrm{M} 3 \mathrm{G}$ & $M 6 G$ & Naloxone & Naltrexone & $\begin{array}{c}\text { Target conc. } \\
\mu g / L\end{array}$ & BUP & NBUP \\
\hline \multirow{6}{*}{$\begin{array}{c}\text { Trueness } \\
\text { Relative bias }(\%)\end{array}$} & 5.0 & .9 .46 & -7.47 & 13.78 & 1.33 & 7.11 & 13.64 & 0.5 & 15.11 & 10.10 \\
\hline & 10.0 & -13.23 & -10.2 & -4.80 & -7.25 & -4.33 & 2.39 & 1.0 & -9.35 & -6.12 \\
\hline & 30.0 & -5.99 & -0.11 & -4.63 & 1.23 & 1.65 & -2.42 & 3.0 & -7.69 & 6.91 \\
\hline & 150.0 & -6.95 & 3.51 & -2.13 & -0.41 & 2.66 & 0.05 & 15.0 & -3.64 & -8.14 \\
\hline & 300.0 & -1.90 & 3.85 & 3.39 & -3.01 & -1.67 & 3.22 & 30.0 & 2.61 & 2.16 \\
\hline & 500.0 & -2.17 & -1.94 & -0.42 & -3.21 & 7,27 & 0.57 & 50.0 & -4.97 & -3.84 \\
\hline \multirow{6}{*}{$\begin{array}{c}\text { Intra-assay preci- } \\
\text { sion } \\
\text { Repeatability } \\
\text { (RSD\%) }\end{array}$} & 5.0 & 10.36 & 13.54 & 11.94 & 20.91 & 11.95 & 13.54 & 0.5 & 9.81 & 34.70 \\
\hline & 10.0 & 5.96 & 6.28 & 6.55 & 5.93 & 5.89 & 2.95 & 1.0 & 7.84 & 11.18 \\
\hline & 30.0 & 4.09 & 6.69 & 3.47 & 9,43 & 6.00 & 7.96 & 3.0 & 8.80 & 9.46 \\
\hline & 150.0 & 6.56 & 5.11 & 6.66 & 5.85 & 6.89 & 7.82 & 15.0 & 6.11 & 8.13 \\
\hline & 300,0 & 3.01 & 6.02 & 4.36 & 7.04 & 6.11 & 5.74 & 30.0 & 5.75 & 7.57 \\
\hline & 500.0 & 5.86 & 4.85 & 5.97 & 5.14 & 3.32 & 2.36 & 50.0 & 5.76 & 9.50 \\
\hline \multirow{6}{*}{$\begin{array}{c}\text { Inter assay preci- } \\
\text { sion } \\
\text { intermediate pre- } \\
\text { cision (RSD\%) }\end{array}$} & 5.0 & 19.53 & 17.28 & 26.77 & 20.91 & 21.25 & 24.01 & 0.5 & 33.91 & 37.34 \\
\hline & 10.0 & 6.43 & 7.95 & 10.96 & 7.14 & 8.96 & 8.60 & 1.0 & 12.12 & 20.63 \\
\hline & 30.0 & 5.51 & 6.74 & 4.27 & 13.75 & 6.48 & 8.91 & 3.0 & 8.80 & 9.46 \\
\hline & 150.0 & 7.15 & 5.42 & 7.36 & 7.05 & 6.89 & 10.46 & 15.0 & 6.11 & 8.44 \\
\hline & 300.0 & 3.01 & 6.02 & 4.36 & 8.86 & 6.16 & 6.61 & 30.0 & 5.75 & 7.57 \\
\hline & 500.0 & 5.86 & 6.32 & 6.78 & 5.98 & 3.32 & 3.13 & 50.0 & 8.04 & 9.50 \\
\hline \multirow{6}{*}{$\begin{array}{c}\text { Uncertainty } \\
\text { Relative expended } \\
\text { uncertainty }(\%)\end{array}$} & 5.0 & 44.04 & 37,82 & 60.79 & 44.08 & 47.85 & 54.06 & 0.5 & 77.90 & 79.79 \\
\hline & 10.0 & 13.75 & 17.38 & 24.56 & 15.52 & 19.93 & 19.65 & 1.0 & 27,14 & 46.75 \\
\hline & 30.0 & 12.13 & 14.23 & 9.29 & 30.49 & 13.85 & 19.15 & 3,0 & 18.67 & 20.06 \\
\hline & 150.0 & 15.32 & 11.56 & 15.79 & 15.38 & 14.62 & 23.01 & 15.0 & 12.87 & 18.16 \\
\hline & 300.0 & 6.35 & 12.77 & 9.19 & 19.63 & 13.00 & 14.28 & 30.0 & 12.13 & 16.07 \\
\hline & 500.0 & 12,36 & 13.85 & 14.61 & 12.98 & 6.99 & 6.89 & 50.0 & 17.76 & 20.16 \\
\hline $\begin{array}{l}\mathrm{LQL}(\mu \mathrm{g} / \mathrm{L}) \\
\mathrm{UQL}(\mu \mathrm{g} / \mathrm{L})\end{array}$ & & $\begin{array}{c}5.0 \\
500.0\end{array}$ & $\begin{array}{c}5.0 \\
500.0\end{array}$ & $\begin{array}{c}6.9 \\
500.0\end{array}$ & $\begin{array}{c}5.0 \\
500.0\end{array}$ & $\begin{array}{c}5.0 \\
500.0\end{array}$ & $\begin{array}{c}6.4 \\
500.0\end{array}$ & & $\begin{array}{c}0.8 \\
50.0\end{array}$ & $\begin{array}{c}0.8 \\
50.0\end{array}$ \\
\hline
\end{tabular}

Legend: $M E T H A=$ methadone, $M 3 G=$ morphine-3-glucuronide, $M 6 G=$ morphine 6 -glucuronide, $B$ UP $=$ buprenorphine, $N B U P=$ norbuprenorphine. 

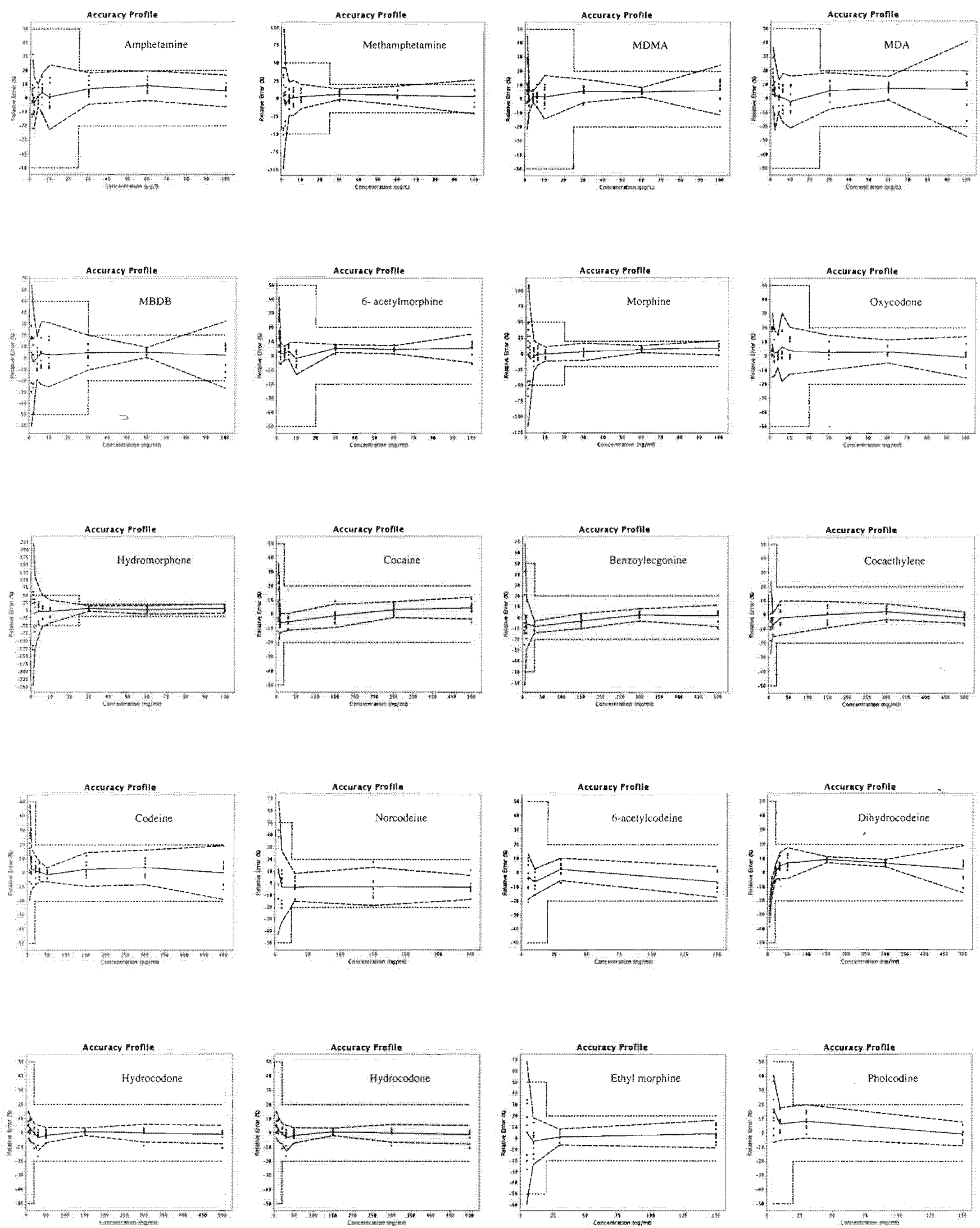

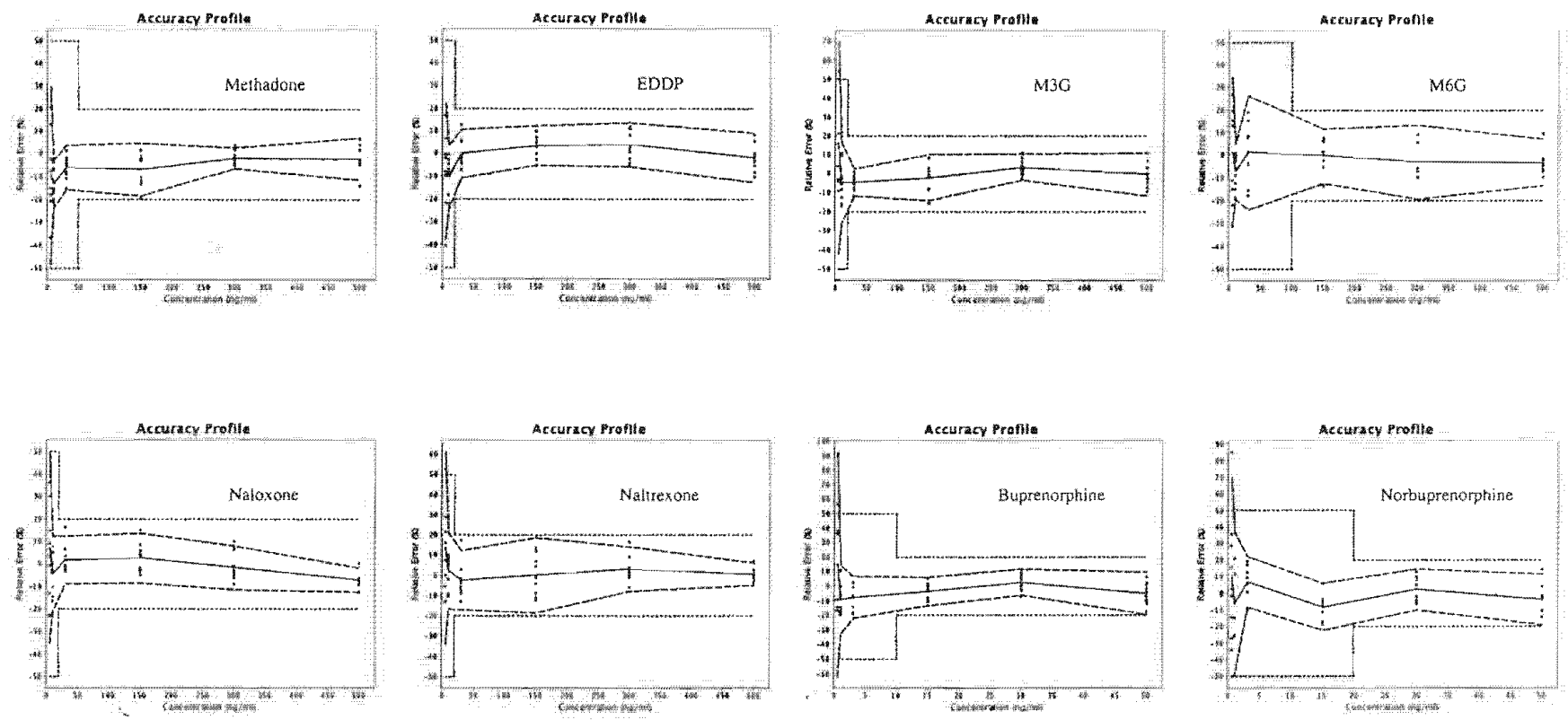

Figure 2-Accuracy profiles for each analyte

Legend: The plain line is the relative bias, the dashed lines are the $\beta$-expectation tolerance limits and the dotted curves represent the acceptance limits (20\%). The dots represent the relative back-calculated concentrations and are plotted with respect to their targeted concentration.

measurand. The expanded uncertainty represents an interval around the results where the unknown true value can be observed with a confidence level of $95 \%$. The relative expanded uncertainties (\%) are obtained by dividing the corresponding expanded uncertainties with the corresponding introduced concentrations. Values for each analyte are presented in Table 2.

The total error evaluates the ability of the method to produce accurate results. Thus, the total error estimation of a procedure is fundamental to assess the validity of the method. Total error is the sum of trueness and precision, and is clearly a good indicator of results accuracy. The accuracy expresses the closeness of agreement between the value found and the value which is accepted either as a conventional true value or an accepted reference value $(12,13)$.

The accuracy profile is obtained by joining the extremes of the $87.5 \%$ interval, i.e. the interval that will contain $87.5 \%$ of the future individual results. The acceptance limits were set at $\pm 20 \%$ for concentrations upper than $20 \mu \mathrm{g} / \mathrm{L}$ and at $\pm 50 \%$ for concentrations

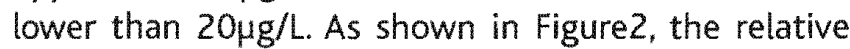
upper and lower $\beta$-expectation tolerance intervals did not exceed the acceptance limits for each compound in the dosing range.

The intersections between the accuracy profile and the acceptance limits define the lower limit of quanti- tation (LQL) as well as the upper limit of quantitation (UQL) $(12,13)$. LQL and UQL of all compounds are presented in Tablez.

\section{DISCUSSION}

Sample pre-treatment was fairly rapid; it consisted in a simple solid-phase extraction procedure. Contrary to GC-MS method, UHPLC method did not require derivatization of the sample which is time-consuming. Glucuronide conjugated compounds of morphine were monitored in the method, therefore hydrolysis step was not necessary anymore. We were not able to validate heroin analysis with this method; actually, heroin is not stable at room temperature, so sample had to be analysed as rapidly as possible; furthermore, it is not stable at basic $\mathrm{pH}$, elution step during SPE should be adapted (9). The use of an Acquity HSS T3 column allowed separating efficiently polar compounds (notably morphine-6-glucuronide and morphine-3-glucuronide) contrary to a classical C18 column. UHPLC technology allowed to obtain very good and reproducible chromatographic separation in a fairly short time, it was important to have a separation as good as possible to be able to measure two MRM by analyte in the mass spectrometer ; indeed, only 19 MRM channels can be 
measured by time function. The MS method was divided in five time functions and MRM dwell times were adjusted to maximize sensitivity.

The quantitative determination of cocaine, opiates and amphetamines in serum answered to our objectives : function responses were established for each analyte, method presented an acceptable linearity : from LQL to $500 \mu \mathrm{g} / \mathrm{l}$ for cocaine, benzoylecgonine, cocaethylene, codeine, dihydrocodeine, methadone, EDDP, M3G, M6G, naloxone and naltrexone; from LQL to $300 \mu \mathrm{g} / \mathrm{L}$ for norcodeine; from LQL to $150 \mu \mathrm{g} / \mathrm{L}$ for 6-acetylcodeine, ethylmorphine and pholcodine, from $\mathrm{LQL}$ to about $100 \mu \mathrm{g} / \mathrm{L}$ for amphetamine, methamphetamine, MDMA, MDA, MBDB, 6-acetylmorphine, morphine, oxycodone and hydromorphone; from LQL to $50 \mu \mathrm{g} / \mathrm{l}$ for buprenorphine and norbuprenorphine. Biases were smaller than $19 \%$ for concentrations lower than $20 \mu \mathrm{g} / \mathrm{L}$ and than $12 \%$ for concentrations upper than $20 \mu \mathrm{g} / \mathrm{L}$. For repeatability, RSDs did not exceed $20 \%$ for concentrations lower than $20 \mu \mathrm{g} / \mathrm{L}$ and $8 \%$ for concentrations upper than $20 \mu \mathrm{g} / \mathrm{L}$; they did not exceed $30 \%$ for concentrations lower than $20 \mu \mathrm{g} / \mathrm{L}$ and $15 \%$ for concentrations upper than $20 \mu \mathrm{g} / \mathrm{L}$ for intermediate precision. The lower and upper $\beta$-expectation tolerance limit did not exceed the acceptance limits (20\% for concentrations lower than $20 \mu \mathrm{g} / \mathrm{L}, 50 \%$ for concentrations upper than $20 \mu \mathrm{g} / \mathrm{L}$ ) at $87.5 \%$ level. The lower limit of quantitation was lower than $7 \mu \mathrm{g} / \mathrm{L}$ for each compound which was quiet better than LQL generally obtained by GC-MS technology.

\section{CONCLUSION}

In order to detect easily and rapidly narcotic substances responsible for addiction and/or intoxication, we developed a method which allowed quantifying simultaneously 27 compounds belonging to cocaine, opiates or amphetamines chemical family in serum by UHPLC-MS/MS. This method is fully validated using total error approach which is a really innovative procedure for analytical validation in toxicological laboratories. Finally, this method could be used as a generic method to identify and quantify ones of the most common drugs of abuse in human serum.

\section{REFERENCES}

1. Observatoire européen des drogues et des toxicomanies, Rapport annuel, Luxembourg, Office des publications de l'Union Européenne, 2009.

2. Jagerdeo E., Montgomery M.A., LeBeau M.A., Sibum M. An automated SPE/LC/MS/MS method for the analysis of cocaine and metabolites in whole blood. 1 of Chrom $B, 2008 ; 874: 15-20$.

3. Chèze M., Deveaux M., Martin C., Lhermitte M., Pépin C., Simultaneous determination of six amphetamines and analogues in hair, blood and urine by LC-ESI-MS/MS. Application to the determination of MDMA after low ecstasy intake. Forensic Sci int. 2007; 170: 100-104.

4. Bjork M.K., Nielsen M.K.K., Markussen L.O., Klinke H.B., Linnet K. Determination of 19 drugs of abuse and metabolites in whole blood by high-performance liquid chromatography-tandem mass spectrometry. Anal Bioanal Chem, 2009, in press.

5. Sergi M., Bafile E., Compagnone D., Curini R., D'Ascenzo G., Romolo F.S., Multiclass analysis of illicit drugs in plasma and oral fluids by LC-MS/MS. Anal Bioanal Chem, 2009; 393: 709m718.

6. Lacroix C., Saussereau E., Bodin $G$., Coulle J.P. Quantification des opiacés, cocainiques et amphétamines par chromatographie liquide haute performance/spectrométrie de masse en tandem après préparation en ligne de l'échantillon. ATA, 2008; 20(1): $25-38$

7. Paterson 5., Lintzeris N., Mitchell T.B., Cordero R., Nestor L., Strang I., Validation of techniques to detect illicit heroin use in patients prescribed pharmaceutical heroin for the managem ment of opioid dependence. Addiction, 2005; 100: 1832-1839.

8. Kudo K., Ishida T, Hara K., Kashimura S., Tsuil A., Ikeda N., Si multaneous determination of 13 amphetamine related drugs in human whole blood using an enhanced polymer column and gas chromatography-mass spectrometry. J of Chrom B. 2007; 855: 115-120.

9. Rook E.J., Hillebrand M.J., Rosing H. et al. The quantitative analysis of heroin, methadone and their metabolites and the simultaneous detection of cocaine, acetylcodeine and their metabolites in human plasma by high-performance liquid chromatography coupled with tandem mass spectrometry. $/$ of Chrom 8, 2005; 824:213-221.

10. Hubert Ph., Nguyen.Huu J.- -., Boulanger B . et al. Harmonization of strategies for the validation of quantitative analytical procedures. A SFSTP proposal - Part 1. J. Pharm Biomed Ana. 2004; 36:579-586.

11. Hubert Ph., Nguyen-Huu J.-J., Boulanger B . et al. Harmonization of strategies for the validation of quantitative analytical procedures. A SFSTP proposal - Part II. I Pharm Biomed Anal 2007; 45:70m81.

12. Hubert Ph., Nguyen-Huu J." .. Boulanger B . et al. Harmonization of strategies for the validation of quantitative analytical procedures. A SFSTP proposal - Part II. J Pharm Biomed Anal 2007; 45: 82-96.

13. Rozet E., Ceccato A., Hubert C. et al., Analysis of recent pharmaceutical regulatory documents on analytical method validation, J of Chrom A, 2007, 1158, 111-125. 